\title{
A inserção do Profissional de Educação Física nos Núcleos de Apoio a Saúde da Família: visão dos profissionais
}

\author{
Physical Educational Professionals insertion on the Family Health Center \\ of Support: professional's vision
}

\author{
Talitha Bonini Ferreira', Mariana Dantas Cipolotti', Bruna Gabriela Marques', Maria Luiza de Jesus Miranda
}

\section{Resumo}

Criado em 2008, o Núcleo de Apoio à Saúde da Família (NASF) surgiu com a finalidade de aumentar as ações da Estratégia de Saúde da Família (ESF) por meio de sua atuação integrada com as equipes de saúde e todas as redes de serviço. O objetivo deste estudo foi analisar a percepção de Profissionais de Educação Física (PEF) inseridos no NASF sobre as funções e a efetividade da intervenção multiprofissional nas equipes no Município de São Paulo. Participaram do estudo oito profissionais com experiência no NASF (atualmente inseridos no NASF ou que já trabalharam nessas equipes). Foi utilizada uma entrevista semiestruturada para obter as informações, analisadas por meio de análise de conteúdo. Observou-se que os participantes trouxeram informações positivas a respeito do NASF, mostrando como são realizadas as atividades e quais suas funções no grupo. Os resultados apresentados por esta pesquisa apontam que a inserção do PEF é relevante nas equipes NASF, pois representam uma área de conhecimento e intervenção habilitada para desenvolver ações e programas de educação em saúde, sendo a promoção à atividade física $e$ modos de vida saudáveis, parte constituinte deste processo. Porém, foram demonstrados lacunas e desafios a serem enfrentados por PEF no município de São Paulo para a consolidação da sua inserção nessas equipes.

\section{Palavras-chave}

Profissional da saúde; Equipes multidisciplinares; Pesquisa qualitativa; Profissional de Educação Física.

\begin{abstract}
The Family Health Support Nucleus (Núcleo de Apoio à Saúde da Familia - NASF) was created in 2008 in order to increment the actions of the Family Health Strategy (Estratégia de Saúde da Familia-ESF) through its integrated co-operation with the healthcare teams and all services networks.. The objective of this study was analyzing the perception of PEF inserted into NASF on the functions and effectiveness of multiprofessional intervention teams in the city of São Paulo. The participants of the study were eight professionals with NASF experience (have worked or currently work in these teams). A semi-structured interview was used to obtain the information, subsequently analyzed through the content analysis methodology. The results show that the professionals positive information about their positions there and the activities of the Nucleus. Also as a result of this research we found that the placement of PEP in the NASF's teams is relevant as they represent a field of knowledge and intervention qualified to develop actions and programs in bealth education, being the promotion of the physical activity and healthy life styles a part of this process. However, gaps were found and there are challenges to be faced by PEP in the city of São Paulo in order to consolidate their placement in these teams.
\end{abstract}

\section{Keywords}

Health Professional; Multi-disciplinary Teams; Qualitative Research; Physical Education Professional.

\section{Introdução}

Com o propósito apoiar a consolidação da Atenção Primária à Saúde/Estratégia da Saúde da Família (APS/ ESF), em 2008 o Ministério da Saúde (MS) criou

\footnotetext{
1 Universidade São Judas Tadeu. São Paulo, SP. Brasil.
}

os Núcleos de Apoio à Saúde da Família (NASF) ${ }^{1}$, buscando ampliar a abrangência, a resolubilidade, a territorialização, a regionalização e a ampliação das ações da Atenção Básica no cuidado em saúde. Esta reorientação nas políticas públicas brasileiras tem como eixo norteador de suas ações e diretrizes a abordagem multicausal no processo saúde-doença, que 
compreende a determinação do estado de saúde de uma pessoa como um processo complexo, proveniente de fatores sociais, econômicos, culturais, étnicos/raciais, psicológicos e comportamentais, inter-relacionados e dinâmicos, que influenciam a ocorrência de problemas de saúde e seus fatores de risco na população.

Nesse sentido, para que as intervenções ampliem seu escopo de modo a organizar a atenção e o cuidado na articulação com uma equipe multiprofissional, as ações devem ser definidas pelos gestores municipais e as equipes de ESF mediante critérios de pioridades identificadas a partir das necessidades do território e deverão seguir um modelo de implantação específico ${ }^{1,2}$.

Conforme a portaria 3.124/GM de 2012, poderão compor as o NASF as seguintes ocupações referidas no Código Brasileiro de Ocupações: Médico Acupunturista; Assistente Social; Profissional/Professor de Educação Física; Farmacêutico; Fisioterapeuta; Fonoaudiólogo; Médico Ginecologista/Obstetra; Médico Homeopata; Nutricionista; Médico Pediatra; Psicólogo; Médico Psiquiatra; Terapeuta Ocupacional; Médico Geriatra; Médico Internista (clinica médica), Médico do Trabalho, Médico Veterinário, profissional com formação em arte e educação (arte educador) e profissional de saúde sanitarista, ou seja, profissional graduado na área de saúde com pós-graduação em saúde pública ou coletiva ou graduado diretamente em uma dessas áreas ${ }^{3}$.

Aos profissionais corresponde a responsabilidade de promover ações e serviços que considerem os determinantes e condicionantes da saúde e, ao mesmo tempo, promover práticas educativas que contemplem as condições de vida, com o propósito de conscientizar os usuários e a comunidade para escolhas e condições de vida saudáveis ${ }^{1,2}$.

Salienta-se a expansão significativa das equipes de NASF implantadas no território brasileiro, as quais somam 4.444 equipes de NASF, referentes à modalidade 1,2 e 3 e as equipes Intermunicipais. Deste total, 345 equipes encontram-se no Estado de São Paulo, sendo 225 equipes de NASF1, 38 equipes de NASF2, 80 equipes de NASF3 e 2 NASFs Intermunicipais. Para o município de São Paulo, percepe-se uma clara redução no número e nas modalidades de NASFs, encontram-se em funcionamento $72 \mathrm{NASF}$ do tipo $1^{4}$.

Segundo dados do MS os núcleos beneficiam 94,8\% dos municípios brasileiros, com mais de 31.400 equipes implantadas. Santos apontava, em 2012, que do total de equipes do NASF, 49,2\% possuiam o profissional de Educação Física PEF nos Núcleos 5 .

No município de São Paulo, a organização da gestão de saúde difere de outros municipios do país, uma vez que a ESF e os NASF são gerenciados por instituições coparceiras da Secretaria Municipal de Saúde. São instituições privadas contratadas pela prefeitura e desempenham as funções de coordenação e supervisão, bem como realizam a contratação e treinamento dos profissionais da atenção básica ${ }^{6}$.

Segundo Carvalho et. al. ${ }^{7}$, o papel do PEF no NASF é coordenar, planejar, realizar treinamentos especializados, participar de equipes multidisciplinares e interdisciplinares, entre outros. Há um enorme campo de atuação no NASF a ser ocupado pelos PEF, após a recente revisão da Política Nacional de Atenção Básica que atribuiu de forma integrada o profissional do NASF à Rede de Atenção à Saúde e seus serviços, a exemplo do Centro de Atenção Psicossocial (CAPS), Centro de Referência em Saúde do Trabalhador (CEREST), ambulatórios especializados, entre outras redes ${ }^{3}$.

Diante do exposto, o objetivo desse estudo foi analisar a percepção de PEF inseridos no sobre as funções e a efetividade da intervenção multiprofissional nas equipes do Município de São Paulo. 


\section{Métodos}

Esse estudo foi desenvolvido numa abordagem qualitativa do tipo descritivo exploratório, conforme apontam Lakatos e Marconi ${ }^{8}$.

Os participantes da pesquisa foram selecionados por meio de indicações dos profissionais entrevistados, estratégia coerente com o método de amostragem baseado em cadeia de referências, ou "bola de neve". ${ }^{9,10}$ Nesse sentido, foi solicitado a cada profissional a indicação de algum outro colega para ser contatado que atendesse ao critério experiência atual ou passada no NASF. O primeiro entrevistado do NASF foi indicação de um fisioterapeuta de Unidade Básica de Saúde (UBS), colega de trabalho de uma das pesquisadoras.

Para se definir o tamanho da amostra levou-se em consideração o saturamento de informações obtidas. Foram oito profissionais formados em Educação Física de ambos os sexos, cinco mulheres e três homens, com idades entre 30 e 45 anos, média $34 \pm 5,29$, todos do Município de São Paulo, participantes com experiência no NASF.

O caráter de participação na pesquisa foi voluntário e aqueles que concordaram em participar do estudo assinaram o Termo de Consentimento Livre e Esclarecido. O projeto de pesquisa foi aprovado pelo Comitê de Ética e Pesquisa da Universidade São Judas Tadeu (parecer $n^{\circ}$ 235.909).

Os participantes responderam a uma entrevista semiestruturada, envolvendo uma série de perguntas abertas em uma ordem prevista, mas quando necessário, o entrevistador pode acrescentar perguntas de esclarecimento ${ }^{11}$. A coleta das informações ocorreu entre os meses de julho a setembro de 2013, em dias e horários previamente agendados e realizada em locais indicados pelos participantes. As entrevistas duravam em média 60 minutos e as respostas foram gravadas e, posteriormente, transcritas. Procedeu-se à leitura de cada entrevista de forma individual para identificar as unidades de significado e, após esta fase, foi possível identificar padrões de respostas dentre os depoimentos, os quais permitiram a elaboração de eixos temáticos. A interpretação dos resultados foi conduzida por uma abordagem qualitativa e, devido às intenções da pesquisa, os resultados foram apresentados descritivamente ${ }^{12,11 .}$

A apresentação dos resultados foi organizada em categorias, a partir da perspectiva dos PEF, manifestada por meio das respostas às questões da entrevista.

\section{Resultados}

Todos os sujeitos que foram convidados a participar da pesquisa, aceitaram. Dessa forma, não houve recusas ou perdas de participantes para o estudo. A seguir são apresentados os resultados, segundo as categorias observadas.

\section{"O conhecimento a respeito do Núcleo"}

Ao relatarem sobre como conheceram o NASF, observamos que um participante teve conhecimento antes da implantação da equipe, pois foi "acompanhando a própria elaboração da política"(PEF1), enquanto alguns o conheceram através de contatos profissionais e, outros, apenas por meio da divulgação dos editais de seleção para vagas nessas equipes. A contratação e inserção de todos os entrevistados se deu por meio de aprovação em processo de seleção específico para esta atuação.

\section{"Percepção das funções do NASF"}

Quando se questionou sobre a atuação específica do PEF no NASF, cada sujeito apontou diversas funções que esse profissional pode assumir na equipe. Dentre essas destacam-se: ministrar sessões de atividade física e exercício físico, realizar 
avaliação física, orientar para hábitos saudáveis, trabalhar na prevenção de doenças, ampliar o campo de pesquisa, auxílio na saúde mental, melhora do desempenho e trabalho social. Além destas funções, os sujeitos ressaltaram as reuniões em equipe, as visitas domiciliares, as relações interpessoais, o compartilhamento de atividades, articulação com a comunidade/rede de serviço e o matriciamento, o cumprimento de metas de outras equipes do SUS e a quebra de preconceitos.

Para ilustrar as funções atribuídas pelos participantes, destacamos alguns trechos das entrevistas,

"[...] trabalhávamos com grupos de pessoas (grupo de caminhada, grupo de terceira idade, grupo de crianças com alguns esportes), realizando atividades dentro da própria unidade ou em parcerias na região: parques, igrejas, escolas..."(PEF3).

"[...]participação em reuniões de equipe, discussão de todos os tipos de caso ... realização de visita domiciliar ... nas unidades que agente participava tinha alguns grupos já de promoção e prevenção...”(PEF4).”

"...As funções do NASF estão ligadas à atenção integral e sempre com o apoio da ESF..." (PEF6)

Também foi citado por vários participantes que no começo do programa os próprios profissionais não sabiam o que era para ser feito, como ilustra o trecho a seguir:

"As funções... Isso não tava bem claro, não existia..." (PEF1.)

Quando questionados sobre sua visão em relação ao NASF, apenas dois apresentaram uma opinião negativa sobre o Núcleo:

"...quando chega na realidade, a gente tem um sistema de saúde que é completamente subfinanciado... e a gente acaba cumprindo um papel de enxugar gelo e tapar buraco ..."(PEF1).

"Eu acho que nunca vai chegar a ser do jeito que eles querem"(PEF2).

Os demais, apresentaram uma visão positiva, como pode-se perceber nos seguintes trechos:

"O NASF veio para acrescentar e apoiar o Programa Saúde da Familia”(PEF3).

"O NASF é altamente rico em termos de troca..."(PEF4).

\section{"Percepção do trabalho nas equipes multiprofissionais"}

A percepção dos PEF inseridos no NASF do Município de São Paulo, a respeito do trabalho multiprofissional e sua efetividade chamam a atenção pelas respostas díspares, relacionadas à proposta de intervenção eficiente, dificuldades na organização e articulação de processos de trabalho com os demais profissionais da área da saúde e predomínio da visão do médico, como exemplificado nos trechos a seguir:

"No começo era um pouco difícil, por causa do preconceito que, como educador físico eu sofria. Principalmente dos médicos. Alguns tinham mania de achar que sabiam tudo[...] (PEF1)".

"[...] as pessoas da equipe não tinham comprometimento, não iam na reunião, os enfermeiros e médicos principalmente..."(PEF2).

Ao serem questionados se as equipes garantiam bons resultados, parte dos entrevistados apontou que o bom funcionamento de uma equipe se dá pela boa relação entre seus membros e o comprometimento da equipe com a perspectiva do cuidado em saúde. Os trechos a seguir ilustram isso: 
"Com certeza [...] a desmistificação de ideias, o relacionamento, a melhora da saúde das pessoas... essas coisas, são bons resultados"(PEF1).

"Eu trabalhei em duas equipes diferentes, na mesma região. Uma demorou a dar bons resultados porque as pessoas da equipe não tinham comprometimento [...] Na outra equipe as pessoas estavam mais afim de fazer aquilo acontecer, a equipe tentava, era diferente a dinâmica. Então acho que varia muito de unidade para unidade" (PEF 2).

O matriciamento foi também apontado por alguns integrantes do GNASF como um fator que contribui para com bons resultados, ilustrado pelo trecho abaixo:

"[...]pelo menos a minha equipe é fantástica, a gente se matricia, então nós nos capacitamos pra lidar com diversas situações [...]"(PEF8).

"Dificuldades na atuação profissional"

"[...]O trabalho multidisciplinar é um grande desafio, pois ao mesmo tempo que existe a troca de saberes e conhecimento, existe os conflitos de ideias[...]"(PEF3).

Mesmo tendo a percepção de bom funcionamento dessas equipes, foram indicados problemas que atrapalharam a geração de resultados. Os relatos aqui apresentados demonstram as lacunas e os desafios apontados por PEF que atuam no NASF no Muncipio de SP:

"No começo era um pouco difícil, por causa do preconceito que, como educador físico eu sofria. Principalmente dos médicos. Alguns tinham mania de achar que sabiam tudo, que dominavam tudo"(PEF1).

"[...] muitos dos problemas estão na infraestrutura, não tem com o que trabalhar, onde trabalhar... mesmo sendo uma unidade grande eu teria que trabalhar na rua... ter parcerias intersetoriais com igrejas, escolas, parques porque a gente não tem local mesmo[...]( PEF3).

Quando se questionou como era trabalhar na equipe e se os profissionais gostavam de estar inseridos na equipe, um participante apontou um fator negativo:

"[...] en gostava por ser um trabalho diferente... mas o que empacava eram sempre os mesmos problemas... a gente tinha vários chefes... cada gerente pedia que a gente fizesse uma coisa, então empacava o serviço, a gente sabia que não podia fazer certas coisas, e o gerente pedia para que a gente fizesse"(PEF 2).

Os outros participantes apontaram fatores positivos, como podemos ver nos trechos a seguir:

"Muito bom. Tinhámos algumas divergências como em toda equipe com mais de uma cabeça pensante, mas era muito bom. Bem efetivo"(PEF1).

"Era uma atividade muito gratificante e realmente o trabalho era quase $100 \%$ em equipe. [...] gostava porque era algo em que eu aprendia muito[...]”(PEF3).

"É rico e desafiador assim, eu acho que assim: a equipe que eu trabalho é super acolhedora, os profissionais são super capacitados, assim muito envolvidos, uma disponibilidade pra discutir, pra tá junto, dentro do possível o máximo que pode"(PEF4).

\section{Discussão}

Sabe-se que o trabalho realizado no NASF é feito de forma multiprofissional, podendo ser definida como "[...conjunto de relações e interações que acontecem entre profissionais que trabalham juntos...]"13, sendo ressaltado por Scabar, Peli- 
cioni e Pelicioni ${ }^{14}$, que dentro dessas equipes, cada profissional tem o seu papel e especialidade. Assim há um comprometimento com a integralidade nas propostas de promoção da saúde, que não abordam o indivíduo de forma fragmentada, ou mesmo, restrita ao funcionamento dos sistemas fisiológicos.

Com a Portaria no 154, de 24 de janeiro de 2008, já citada, estão relatadas quais funções que o PEF deve assumir na equipe multiprofissional. Além de ministrar sessões de atividade física, deve estar apto para veicular informações, incentivar a criação de espaços para inclusão social, proporcionar educação permanente em atividade física, nutrição e saúde, articular parcerias, capacitar profissionais (principalmente os ACS), entre outras atividades.

Embora a maioria dos entrevistados tenha considerado como principal função do PEF a promoção da atividade física, ressaltam-se posicionamentos coerentes com a atuação do PEF no NASF, que podem ser elucidadas no estudo de Scabar, Pelicioni e Pelicioni ${ }^{14}$, no qual o principal foco da intervenção do PEF direcionase para prevenção, promoção, proteção e reabilitação da saúde, demonstrando a coerência e ênfase da atuação profissional sobre os determinantes sociais da saúde, seja no âmbito individual ou territorial ${ }^{14}$. Os autores também incluem como especificidades dos PEF o trabalho com a gestão, políticas de saúde, diagnóstico, planejamento e intervenção na área de práticas corporais e atividades físicas.

O matriciamento foi elencado pelos participantes do estudo como aspecto relevante da participação nessas equipes do NASF. Essas colocações estão em consonância com Cunha e Campos ${ }^{15}$ quando apontam o apoio matricial como sendo ferramenta que oferece apoio assistencial e suporte técnico-pedagógico às equipes e promove o compartilhamento dos saberes, ampliando a resolução dos problemas, com o propósito de corresponsabilização do territorio buscando maior resolutividade para assegurar, de forma dinâmica e interativa os saberes especializados às equipes.

Por outro lado, os relatos dos profissionais inseridos nos NASF do municipio de SP, corroboram com os estudos desenvolvidos por Rodrigues ${ }^{16}$ com os PEF do NASF de João Pessoa- PB e Souza e Loch ${ }^{17}$ com os PEF do NASF no Paraná, ao evidenciar que há um distanciamento do trabalho da Educação Física com as demais profissões em relação à integralidade do cuidado. Os autores apontam que, apesar do reconhecimento da importância do profissinal nas equipes, ainda há um predomínio de ações com grupos específicos pautadas prioritariamente em uma visão biomédica.

Sob o ponto de vista de Campos e Domitti ${ }^{18}$, para alcançar o êxito em trabalhos interdisciplinares é necessário um objetivo comum entre a equipe e a constante disponibilidade ao diálogo e a troca de saberes. Ressaltam que as diferenças existentes entre os profissionais, deverão ser aproveitadas pelos gestores para resolução dos problemas territoriais, na construção dos projetos terapêuticos e nas ações coletivas. Esse ponto de vista é reforçado por Mendes et $a{ }^{19}$ que descrevem a interdisciplinaridade como "a interação dinâmica entre os saberes" e como ponto auxiliar do processo de trabalho e a efetividade do cuidado, na medida em que diferentes conceitos e perspectivas podem interagir e se reconstruirem.

Vale ressaltar que a presença dos PEF na saúde pública ainda é relativamente recente. A revisão sistemática proposta por Neves et al. ${ }^{20}$ assinalou esse aspecto. Os autores afirmam que apenas na década de 1990 intensificaram-se os debates e mobilizações sobre o tema e a importância dos processos educativos na atuação do PEF no campo da Saúde pública. Defendem a proposição de que o grande desafio a ser enfrentado pelo campo da Educação Física é a superação da visão hegemônica e reducionista de promoção da saúde que afeta os processos de intervenção que deveriam primar pelos princípios da integralidade nos processsos de saúde-doença. 
Contudo, os relatos dos PEF do municipio de São Paulo sobre as dificuldades enfrentadas na sua atuação no NASF evidenciam obstáculos de natureza política, vinculadas a estrutura física e organizacional, além de obstáculos de natureza subjetiva, atrelados à cultura e aos aspectos éticos e epistemológicos vinculados a comunicação e relacionamento entre os profissionais das equipes multiprofissionais, fatores também evidenciados na investigação de Campos e Domitti ${ }^{18}$ no municipio de Campinas- SP. Observa-se que, nos dois contextos investigados, as referidas dificuldades atrapalham a efetividade do trabalho multidisciplinarmultiprofisisonais.

Chamam a atenção os obstáculos de natureza política, vinculados às estruturas física e organizacional, por retratarem realidade similar vivenciada por outros profissionais do NASF entrevistados no norte do Paraná ${ }^{19}$ e em Londrina/PR ${ }^{21}$ e em São Paulo ${ }^{22}$, que relataram dificuldades na utilização de salas, materiais e a falta de espaço físico para desenvolvimento das atividades.

Um outro fator atribuido à dificuldade do trabalho no NASF evidenciado pelos entrevistados, está na falta de comprometimento, na não aceitação do trabalho e no preconceito de outros profissionais, fatores também apontados nos relatos dos PEF entrevistados em Londrina ${ }^{21}$.

Um dos temas mais evidenciados pelos PEF, reconhecidos como dificuldades vivenciadas na atuação no NASF, pode ser exemplificado pelo momento que atual do campo da saúde, no qual o modelo tradicional de prestar assistência a saúde vem sofrendo intensas criticas de legitinidade e resolutividade, evidenciando seu esgotamento ${ }^{23}$.

Ayres $^{24}$ enfatiza que as práticas de saúde construidas no bojo das sociedades contemporâneas, vivenciam um momento jamais relatado em sua história, com intensas repercussões e reorientações tanto na formação do profissional de saúde como nos distintos segmentos que compõem o SUS.

Todavia, o estudo sobre as diretrizes do SUS e a saúde coletiva ainda apresentam pouca inserção na organização curricular, em detrimento da concepção hegemônica e mecanicista de conceber a saúde, na qual o corpo é fragmentado e desvinculado de sua subjetividade, traços recorrentes no campo da formação, atuação e pesquisa ainda disseminados pela área, não podemos perder de vista que a demanda contemporânea nas ações em saúde, exige do profissional um fazer interdisciplinar referenciado por compêtencias e habilidades que extrapolam a perspectiva tecniscista ${ }^{25}$.

Soma-se a isso um dos grandes desafios a serem enfrentados por todos os atores envolvidos no sistema: a cultura organizacional no SUS. Nascimento e Oliveira ${ }^{26}$ chamam a atenção para indicadores quantitativos serem o referencial avaliativo da resolutividade e impacto das ações do Sistema. Para os autores, há um equivoco no que tange ao favorecimento do aporte quantitaivo, uma vez que o NASF tem como base de sustenção a clínica ampliada, que visa melhorar a qualidade no cuidado em saúde.

Contudo, não podemos perder de vista, que o NASF é uma nova proposta de trabalho, forjada em seu próprio processo de implantação, na qual os processos de trabalho se constituem na mesma proporção em que a política é implantada, os próprios trabalhadores inseridos nas propostas, do mesmo modo que os demais atores envolvidos - gestores e usuários- vivenciam um processo de assimilação e de apropriação da organização do sistema face ao reconhecimento das necessidades do territorio e o trabalho multiprofissional ${ }^{22}$.

A Educação Física, com suas peculiaridades e áreas de intervenção, deverá responder, necessariamente, as lacunas e desafios decorrentes do campo em formação. Ressalta-se, entretanto, que este movimento deve ser ampliado, com criação 
de novas relações de compromisso e de cooperação entre instituições de ensino superior, Ministérios da Saúde, da Educação, entre outros, efetivando-se assim a inserção do PEF no princípio da integralidade na saúde.

Diante do que obseouvou-se nessa pesquisa, a insersão do PEF nas equipes multiprofissionais se faz relevante pois representa um campo de trabalho em construção. Ressalta-se que a percepção dos profissionais do Municipio de São Paulo sobre suas funções e a efetividade da intervenção multiprofissional é positiva, uma vez que PEF reconhece suas atribuições e funções na equipe, mas relatam que os desafios de sua atuação perpassam a falta de reconhecimento dos outros profissionais no cotidiano das ações do núcleo, estrutura inadequada dos locais de trabalho e a escassez de recursos materiais como um dos grandes desafios a sua atuação.

Outro grande desafio a ser enfretado por todos os envolvidos no sistema, vincula-se as fontes de financiamento do setor saúde, em outras palavras, a expansão da atenção básica, ocorrida com o processo de municipalização, evidenciou um grave problema relacionado aos recursos humanos e financeiros peculiares a cada municipio brasileiro. Ressalta-se que embora exista uma agenda em curso para o aprimoramento do processo de descentralização, este mecanismo deve ser fotalecido e amadurecido com todos os envolvidos no setor.

Uma das possibilidades no enfrentamento dos desafios encontrados pode estar na realização de futuras pesquisas focadas nas relações de trabalho e as demandas do trabalho cotidiano dos núcleos, bem como a análise da sua atuação nas equipes multiprofissionais, especialmente do NASF.

Consideramos como as principais limitações do estudo: a) a constituição da amostra, que poderia ser mais abrangente ou, até, incluir outros profissionais integrantes das equipes, cujas opiniões poderiam proporcionar uma outra visão sobre a inserção e a atuação do PEF; b) um aspecto inerente à pesquisa qualitativa em si, cujos resultados são vinculados ao grupo pesquisado, não podendo ser generalizados diretamente.

A partir dessa pesquisa, podemos observar que os PEF possuem um conhecimento sobre o seu papel a desempenhar nas equipes multiprofissionais, porém, as limitações, desafios devem ser considerados e levados em conta, como um ponto de partida para melhorias no desempenho dos profissionais e também um estímulo para novas pesquisas sobre o assunto,pois ainda é escasso.

\section{Contribuição dos autores}

As autoras Talitha Ferreira Bonini e Mariana Dantas Cipolotti desenvolveram o estudo sob orientação da Prof ${ }^{a}$ Dra Maria Luiza de Jesus Miranda e a autora Bruna Gabriela Marques contribuiu com a discussão e revisão do texto.

\section{Referências}

1. BRASIL. Ministério da Saúde. Secretaria de Atenção à Saúde. Departamento de Atenção Básica. Secretaria de Atenção à Saúde, Departamento de Atenção Básica. Brasília: Ministério da Saúde, 2010.

2. Ministério da Saúde. Secretaria-Executiva. Coordenação de Apoio à Gestão Descentralizada. Diretrizes operacionais para os pactos pela vida, em defesa do SUS e de gestão. Ministério da Saúde. Secretaria-Executiva. Coordenação de Apoio à Gestão Descentralizada. Brasília: Ministério de Saúde, 2006.

3. Ministério da Saúde. Secretaria de Atenção à Saúde. Departamento de Atenção Básica. Política Nacional de Atenção Básica / Ministério da Saúde. Secretaria de Atenção à Saúde. Departamento de Atenção Básica. - Brasília: Ministério da Saúde, 2012.110 p.: il. - (Série E. Legislação em Saúde). 
4. Ministério da Saúde. Secretaria de Atenção à Saúde. Departamento de Informática do SUS. Cadastro Nacional de Estabelecimentos de Saúde: Consultas: Equipes. Brasília, DF: Ministério da Saúde, 2015. Disponível em: <http://cnes.datasus. gov.br/Lista_Tot_Equipes.asp>. Acesso em: 19 jan. 2016.

5. Santos SFS. Núcleo de apoio à saúde da família no Brasil e a atuação do profissional de educação física [dissertação de mestrado em Educação Física]. Universidade Federal de Santa Catarina, Centro de Desportos. 2012.

6. Bousquat A, Cohn A, Elias PEA. Implantação do Programa Saúde da Família e exclusão socioespacial no Município de São Paulo, Brasil. Cad. Saúde Pública. 2006; 22:1935-43.

7. Carvalho JCS, Silva PO, Cruz BS, Santo GE. NASF: os professores de Educação Física conhecem? Efdeportes Revista Digital, Buenos Aires. 2010;141:1-7.

8. Marconi MA, Lakatos EM. Fundamentos de metodologia científica. 7. ed. 297 p. São Paulo: Atlas, 2010.

9. Bogdan RC, Biklen SK. Investigação Qualitativa em Educação. Porto: Porto Editora, 1994.

10. Patton, MQ. Qualitative Research and Evaluation Methods. 3rd ed. Thousand Oaks, CA: Sage Publications, 2002.

11. Laville C, Dionne J, Siman LM. A construção do saber: manual de metodologia da pesquisa em ciências humanas. Porto Alegre: Artmed; Belo Horizonte: Ed. da UFMG, 1999.

12. Denzin KN, Lincoln SY. O planejamento da Pesquisa Qualitativa: Teorias e abordagens / Norman K. Denzin, Yvonna S. Linconln; Traduções Sandra Regina Netz. - Porto alegre: Artmed, 2006. 432 p. ; $25 \mathrm{~cm}$.

13. Matuda CG. Cooperação interprofissional: percepções de profissionais da Estratégia Saúde da Família no município de São Paulo (SP). [Dissertação de mestrado em Serviços de Saúde Pública]. São Paulo: Faculdade de Saúde Pública, Universidade de São Paulo, 2012.

14. Scabar TG, Pelicioni AF, Pelicioni MCF. Atuação do profissional de educação física no Sistema Único de Saúde: uma análise a partir da Política Nacional de Promoção da Saúde e das diretrizes do Núcleo de Apoio à Saúde da Família - NASF. J Health Sci Inst. 2012;30(4):411 - 8 .

15. Cunha GT, Campos GWS. Apoio matricial e atenção primária em saúde. Saúde Soc. 2011;20:961-70

16. Rodrigues ZJ, Ferreira DKS, Silva PA, Caminha IO, Farias Junior JC. Inserção e atuação do profissional de educação física na atenção básica à saúde: revisão sistemática. Rev Bras Ativ Fís Saúde. 2013;18 (1):5-15.

17. Souza SC, Loch M. Intervenção do profissional de educação física nos Núcleos de Apoio à Saúde da Família em municípios do norte do Paraná. Rev Bras Ativ Fís Saúde. 2011;16(1):5-10.

18. Campos GWS, Domitti AC. Apoio matricial e equipe de referência: uma metodologia para gestão do trabalho interdisciplinar em saúde. Cad Saúde Pública. 2007;23(2):399-407.

19. Mendes RMJ, Lewgoy AMB, Silveira EC. Saúde e interdisciplinaridade: mundo vasto mundo. Revista Ciência \& Saúde. 2008; 1:24-32.

20. Neves RLR, Antunes PC, Baptista TJR, Assumpção LOT. Educação Física na saúde pública: Revisão Sistemática. R Bras Ci e Mov. 2015;23(2):163-77.

21. Mendonça AM. Promoção da saúde e processo de trabalho dos profissionais de educação física do núcleo de apoio à saúde da família - NASF. [dissertação de mestrado em Saúde Coletiva] Universidade Estadual de Londrina, 2012.

22. Gonçalvez RMA, Lancman S, Sznelwar LI, Cordone NG, Barros GO. Estudo do trabalho em Núcleos de Apoio a Saúde da Família (NASF), São Paulo, Brasil. Rev Bras Saúde Ocup. 2015;40(131):59-74.

23. Ceccim RB, Feuerwerker L. Mudança na graduação das profissões de saúde sob o eixo da integralidade. Cad Saúde Pública. 2004;20(5):1400-10.

24. Ayres JRCM. O cuidado, os modos de ser (do) humano e as práticas de saúde. Saúde e Sociedade. 2004;13(3):16-29.

25. Freitas FF, Carvalho YM, Mendes VM. Educação Física e Saúde: aproximações com a “clinica ampliada”. Rev Bras Ciênc Esporte. 2013;35(3):639-656.

26. Nascimento DDG, Oliveira MAC. Reflexões sobre as competências profissionais para o processo de trabalho nos Núcleos de Apoio à Saúde da Família. Mundo Saúde (Impr.). 2010;34:92-6.

ENDEREÇO PARA CORRESPONDÊNCIA TALITHA BONINI FERREIRA t.bonini.f@uol.com.br
Rua Antonieta Borges Alves, 64

cangaiba, 03734-310, São Paulo-SP.

Tel: (11)98743-6527
RECEBIDO

REVISADO

$31 / 07 / 2014$

$04 / 10 / 2015$

$07 / 11 / 2015$

$11 / 02 / 2016$

$17 / 02 / 2016$ 